\title{
Use of a single-step approach for integrating foreign information into national genomic evaluation in Holstein cattle
}

\author{
A. R. Guarini, ${ }^{1}$ D. A. L. Lourenco, ${ }^{2}$ L. F. Brito,,${ }^{1,3}$ M. Sargolzaei, ${ }^{1,4}$ C. F. Baes, ${ }^{1}$ F. Miglior,${ }^{1,5}$ S. Tsuruta, ${ }^{2}$ \\ I. Misztal, ${ }^{2}$ and F. S. Schenkel ${ }^{1 *}$ \\ ${ }^{1}$ Centre for Genetic Improvement of Livestock, Department of Animal Biosciences, University of Guelph, Guelph, Ontario, Canada N1G 2W1 \\ ${ }^{2}$ Department of Animal and Dairy Science, University of Georgia, Athens 30602 \\ ${ }^{3}$ Department of Animal Sciences, Purdue University, West Lafayette, IN 47907 \\ ${ }^{4}$ Select Sires Inc., Plain City, OH 43064 \\ ${ }^{5}$ Canadian Dairy Network, Guelph, Ontario, Canada N1K 1E5
}

\section{ABSTRACT}

The use of multi-trait across-country evaluation (MACE) and the exchange of genomic information among countries allows national breeding programs to combine foreign and national data to increase the size of the training populations and potentially increase accuracy of genomic prediction of breeding values. By including genotyped and nongenotyped animals simultaneously in the evaluation, the single-step genomic BLUP (GBLUP) approach has the potential to deliver more accurate and less biased genomic evaluations. A single-step genomic BLUP approach, which enables integration of data from MACE evaluations, can be used to obtain genomic predictions while avoiding doublecounting of information. The objectives of this study were to apply a single-step approach that simultaneously includes domestic and MACE information for genomic evaluation of workability traits in Canadian Holstein cattle, and compare the results obtained with this methodology with those obtained using a multistep approach (msGBLUP). By including MACE bulls in the training population, msGBLUP led to an increase in reliability of genomic predictions of 4.8 and $15.4 \%$ for milking temperament and milking speed, respectively, compared with a traditional evaluation using only pedigree and phenotypic information. Integration of MACE data through a single-step approach ( $\left.\operatorname{ssGBLUP}_{\mathrm{IM}}\right)$ yielded the highest reliabilities compared with other considered methods. Integration of MACE data also helped reduce bias of genomic predictions. When using ssGBLUP $_{\mathrm{IM}}$, the bias of genomic predictions decreased by half compared with msGBLUP using domestic and MACE information. Therefore, the reliability and

Received October 9, 2018.

Accepted March 9, 2019.

*Corresponding author: schenkel@uoguelph.ca bias of genomic predictions for both traits improved substantially when a single-step approach was used for evaluation compared with a multi-step approach. The use of a single-step approach with integration of MACE information provides an alternative to the current method used in Canadian genomic evaluations.

Key words: genomic evaluation, multiple acrosscountry evaluation, multi-country genomic information, single-step genomic best linear unbiased predictor

\section{INTRODUCTION}

In dairy cattle breeding programs, the use of sires from international populations has become common since the development of technologies such as frozen semen and embryo transfers (Vandenplas and Gengler, 2015). When breeding programs rely on the use of foreign bulls, predictions will likely be biased if foreign pedigree, performance records, genetic merit, and different genetic levels between base populations in each country are not taken into account for domestic evaluations (Bonaiti and Boichard, 1995). With the implementations of multi-trait across-country evaluation (MACE; Schaeffer, 1994) performed by Interbull (Uppsala, Sweden) and the exchange of sire genotypes, national breeding programs are now able to properly aggregate foreign information into domestic evaluations to increase their training populations and consequently obtain more accurate genomic predictions. In Canada, genomic evaluations have been successfully implemented in Holstein cattle and other dairy breeds through a multi-step genomic BLUP method since 2009 (msGBLUP; Van Doormaal et al., 2009). When MACE information is available, foreign data are added to the evaluations in an extra step after domestic (national) evaluations are performed. Although national breeding programs perform such evaluations routinely, several critical assumptions required by multi-step procedures are not fully met, hence, there are issues as- 
sociated with them. Since genomic selection (GS) was implemented in 2009, selection intensity increased; by using models that do not properly account for the existing pre-selection of imported bulls, predictions become less accurate and more biased (Vitezica et al., 2011).

Milking temperament (MT) and milking speed (MSP) are sex-limited traits where no phenotypic data (domestic or foreign) are available for bulls; therefore, for multi-step genomic approaches, pseudo-phenotypes, [i.e., deregressed estimated breeding values (DD)] have to be used. However, the use of such pseudo-phenotypes could lead to loss of information, reduction in accuracies, and increase in biases (Legarra et al., 2014). Moreover, one assumption for the use of DD is independence. For instance, highly reliable EBV have more contributions from daughters' performance than from relatives; thus, de-regression is expected to have less effect on estimates. On the other hand, de-regression is expected to have a significant effect on EBV with substantially lower reliabilities (i.e., those with more contributions from relatives; Calus et al., 2016). If the model does not properly account for differences in reliabilities and contributions, double-counting of information, and overestimation of reliabilities can occur (Calus et al., 2016; Vandenplas et al., 2017).

Simultaneous use of pedigree, phenotypic, and genomic information in a single-step approach should theoretically overcome problems arising with the use of DD and consequent reduction in accuracies and biases. Legarra et al. (2009), Aguilar et al. (2010), and Christensen and Lund (2010) developed the single-step genomic BLUP (ssGBLUP), a methodology that combines genotypes, pedigree, and phenotypes into a single evaluation. However, because international phenotypic data are not available at the domestic level, the standard ssGBLUP is not able to integrate MACE and domestic data into a joint evaluation. Gianola and Fernando (1986), Quaas and Zhang (2001, 2006), and Legarra et al. (2007) proposed modifications to the traditional BLUP that simultaneously integrates data from foreign evaluations and considers those as priors for calculations of domestic EBV. Gengler and Vanderick (2008) also proposed a feasible alternative to include external evaluations into a national evaluation system through a Bayesian approach, but indicated the need to further improve the method due to its assumptions. These previous studies led to a further extension to ssGBLUP to integrate MACE data into a domestic single-step genomic evaluation, as proposed by Vandenplas et al. (2014) and implemented by Andonov et al. (2017), Vandenplas et al. (2017), and Colinet et al. (2018).
The objectives of this study were to (1) apply a modification of the current ssGBLUP that simultaneously evaluates domestic and external (MACE) information for genomic evaluation of workability traits in Canadian Holstein cattle, and (2) compare reliability and bias of genomic predictions obtained by this methodology to those obtained by multi-step GBLUP approach.

\section{MATERIALS AND METHODS}

\section{Data}

Genotypes and Pedigree. Genotype data consisted of 33,201 Holstein bulls born between 1960 and 2011 generated using the Illumina Bovine SNP50 BeadChip (50K, Illumina Inc., San Diego, CA) or a greater density panel and then imputed to 50K (nonoverlapping SNP). Genotypes were coded as 0,1 , or 2 for calculation of the genomic relationship matrix $(\mathbf{G})$. Genotype quality control excluded monomorphic SNP, SNP, and individuals with call rate lower than $90 \%$, SNP that were out of Hardy-Weinberg equilibrium with very low probability $\left(P\right.$-value $\left.<10^{-6}\right)$ or with minor allele frequency less than 0.05 , and individuals with parentprogeny Mendelian conflicts. After quality control, the number of genotyped animals was 33,196 and the final genotype data set included 40,894 SNP. The pedigree file used for the analysis was generated by tracing the pedigree of animals with records for each trait to 4 generations back, and its size was 4,652,056 animals for MSP and 3,866,576 for MT.

Phenotypes. A total of 3,173,169 records for MSP and 2,519,932 for MT in first-lactation Holstein cows were phenotypes used in the April 2017 genetic evaluation carried by Canadian Dairy Network (CDN, Guelph, Ontario, Canada, www.cdn.ca).

Domestic and $M A C E E B V$. Official relative breeding values (RBV; i.e., domestic traditional, hereafter identified by subscript D) and MACE EBV (subscript $\mathrm{M}$ ) for bulls registered in the CDN database were obtained from the April 2013 genetic evaluation for workability traits of Holstein cows. In Canada, EBV are standardized to RBV with a mean of 100 and standard deviation of 5 . To use MACE EBV in ssGBLUP evaluations, RBV were transformed back to EBV as

$$
\mathrm{EBV}=\frac{\mathrm{SD} \times(\mathrm{RBV}-100)}{5}+\overline{\mathrm{x}},
$$

where SD and $\overline{\mathrm{x}}$ are the actual standard deviation and mean of the trait, respectively. Both traits had a SD of 0.1 and mean of 0 . 


\section{Methods of Evaluation}

This section describes the fundamental concepts behind (1) the standard ssGBLUP, (2) the modification of the standard ssGBLUP for simultaneously analyzing national and international data, (3) the required corrections to avoid double-counting, and (4) the msGBLUP.

Single-Step GBLUP Model. This method uses information from both genotyped and nongenotyped animals simultaneously. The mixed model equations then become

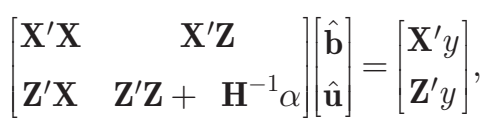

where $\mathbf{y}$ is the vector of trait observations, $\mathbf{b}$ is the vector of fixed environmental effects, $\mathbf{u}$ is the vector of animal additive genetic effects, $\mathbf{X}$ and $\mathbf{Z}$ are the corresponding incidences matrices for $\mathbf{b}$ and $\mathbf{u}, \alpha$ is the variance ratio, and $\mathbf{H}^{-1}$ is derived as in Aguilar et al. (2010) and Christensen and Lund (2010):

$$
\mathbf{H}^{-1}=\mathbf{A}^{-1}+\left[\begin{array}{cc}
0 & 0 \\
0 & \left(\mathbf{G}^{-1}-\mathbf{A}_{22}^{-1}\right)
\end{array}\right]
$$

where $\mathbf{A}^{-1}$ is the inverse of the pedigree relationship matrix, $\mathbf{A}_{22}^{-1}$ is the inverse of the pedigree relationship matrix among genotyped animals, and $\mathbf{G}^{-1}$ is the inverse of the genomic relationship matrix. The $\mathbf{G}$ matrix was constructed as in VanRaden (2008), using observed allele frequencies, where $\mathbf{G}=\mathbf{W W}^{\prime}$, where the elements ij of $\mathbf{W}$ matrix are $w_{i j}=\frac{g_{i j}-2 p_{j}}{\sqrt{2 \sum_{k=1}^{s}\left[p_{k}\left(1-p_{k}\right)\right]}}$, where $g_{i j}$ is the recoded genotype of animal $i$ for SNP marker $j(0,1$, and 2 for genotypes $\mathrm{BB}, \mathrm{AB}$, and $\mathrm{AA}$, respectively) and $p_{j}$ is the frequency of the A allele of SNP $j$, and $s$ is the total number of SNP markers, which is then blended with $5 \%$ of $\mathbf{A}_{22}$ to avoid singularity problems. Based on previous studies (e.g., Misztal et al., 2010), increasing the contribution of polygenic effect in ssGBLUP for dairy cattle evaluations did not improve reliability, whereas bias increased as a greater proportion of $\mathbf{A}_{22}$ was added to $\mathbf{G}$. Lourenco et al. (2018) showed that a polygenic effect greater than $5 \%$ can improve convergence, especially in more complex models, without a considerable effect on reliability and bias.

Integration of External MACE Evaluation. When external information was used, $\mathbf{H}^{-1}$ was adapted from Legarra et al. (2007):

$$
\mathbf{H}^{*-1}=\left[\begin{array}{cc}
\mathbf{T}^{-1}-\mathbf{H}_{\mathrm{MM}}^{-1}+\mathbf{H}^{\mathrm{MM}} & \mathbf{H}^{\mathrm{MD}} \\
\mathbf{H}^{\mathrm{DM}} & \mathbf{H}^{\mathrm{DD}}
\end{array}\right],
$$

where $\mathbf{H}^{\mathrm{MM}}, \mathbf{H}^{\mathrm{MD}}, \mathbf{H}^{\mathrm{DM}}$, and $\mathbf{H}^{\mathrm{DD}}$ are submatrices of the relationship matrix for external, MACE (M), and domestic (D) animals, $\mathbf{H}_{\mathbf{M M}}^{-1}$ is the inverse of the relationship matrix among external animals, and $\mathbf{T}$ is a diagonal matrix with prediction error variance for external animals. The $\mathbf{H}_{11}^{-1}$ part of the Equation [4] should have been calculated using the corresponding elements from the $\mathbf{A}$ matrix instead of the $\mathbf{H}$. However, this was not investigated here as the diagonal elements estimated based on both matrices were expected to be fairly similar (i.e., significant effects on the results are not expected) and due to ease of practical implementation in a ssGBLUP setting. To avoid singularity problems, off-diagonal elements of $\mathbf{H}_{\mathbf{M M}}^{-1}$ are ignored (Legarra et al., 2007). The BLUP90MBE software, a modification of BLUP90IOD2 (Tsuruta et al., 2001), was used to calculate single-step genomic estimated breeding values (ssGEBV) using iteration on data by the method of the preconditioned conjugate gradient. Following Tsuruta et al. (2011), a convergence criterion of $10^{-14}$ was used.

Corrections to Avoid Double-Counting. Consider a national and multi-national evaluation for the same set of individuals with the same pedigree and genotype data, but independent phenotypic data. The aim is to integrate predictions obtained with multi-national evaluation into a national evaluation. One concern is that double-counting of information due to relationships among MACE animals is not taken into account. The EBV of an animal is calculated considering information from its own records as well as records from its parents and offspring. Hence, EBV for an individual that contributed to MACE, when used in national evaluation, leads to information being double-counted (Vandenplas et al., 2014). Gengler and Vanderick (2008) have also investigated this matter using selection index theory and indicated the need for further studies.

In the present study, bulls whose domestic traditional evaluation (i.e., EBV and associated reliability) contributed to MACE EBV were identified based on the nonzero national effective daughter contributions reported by Interbull. A total of 1,729 and 1,788 bulls had MACE EBV for MT and MSP, respectively. Of those, 100 and 109 had nonzero national effective daughter contributions (i.e., bulls whose national information contributed to MACE). Thus, domestic EBV and associated reliabilities for those animals were used and corrections to avoid double-counting of phenotypic 
Table 1. Descriptive statistics for multi-trait across-country evaluation (MACE) EBV used as external information in single-step genomic BLUP for evaluation of milking temperament (MT) and milking speed (MSP)

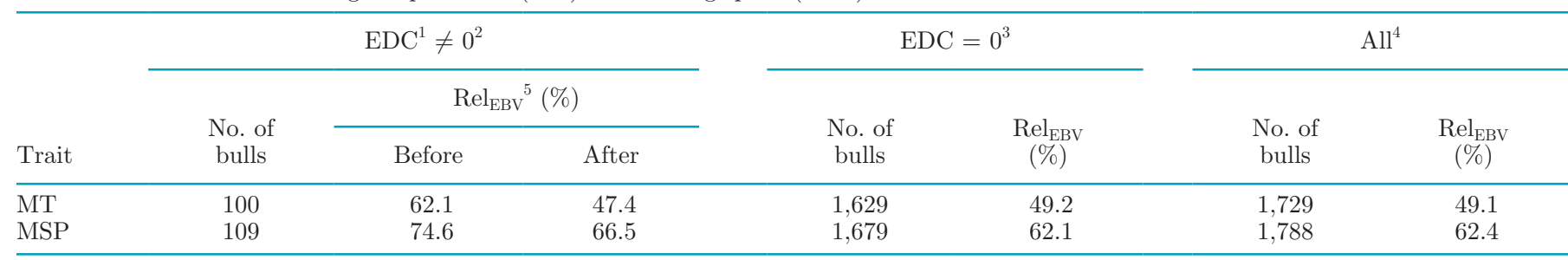

${ }^{1}$ Effective daughter contributions (EDC).

${ }^{2}$ Bulls whose domestic information contributed to MACE.

${ }^{3}$ Bulls whose MACE EBV had no contribution from domestic data.

${ }^{4}$ Total number of MACE bulls included in the analysis.

${ }^{5} \operatorname{Rel}_{\mathrm{EBV}}=$ reliability of EBV before and after corrections to avoid double-counting were applied.

information were applied (see Supplemental File S1; https://doi.org/10.3168/jds.2018-15819), simplifying derivations from VanRaden et al. (2014) and Vandenplas et al. (2014):

$$
\begin{gathered}
\mathrm{EBV}^{*}=\frac{\left[\left(\mathrm{DE}_{\mathrm{M}}+k\right) \times \mathrm{EBV}_{\mathrm{M}}\right]-\left[\left(\mathrm{DE}_{\mathrm{D}}+k\right) \times \mathrm{EBV}_{\mathrm{D}}\right]}{\left(\mathrm{DE}_{\mathrm{M}}-\mathrm{DE}_{\mathrm{D}}\right)+k}, \\
\operatorname{Rel}_{\mathrm{EBV}^{*}}=\frac{\mathrm{DE}_{\mathrm{M}}-\mathrm{DE}_{\mathrm{D}}}{\left(\mathrm{DE}_{\mathrm{M}}-\mathrm{DE}_{\mathrm{D}}\right)+k},
\end{gathered}
$$

where RelEBV* is the reliability of $\mathrm{EBV}^{*}$, and $\mathrm{EBV}^{*}$ are MACE EBV obtained from Interbull $\left(\mathbf{E B V}_{\mathbf{M}}\right)$ after domestic information $\left(\mathrm{EBV}_{\mathrm{D}}\right)$, used to generate foreign $\mathrm{EBV}$, is removed to avoid double-counting; $\mathrm{DE}_{\mathrm{M}}$ and $\mathrm{DE}_{\mathrm{D}}$ are daughter equivalent from foreign and national data, respectively; $k$ is the variance ratio calculated as $\left(4-2 h^{2}\right) / h^{2}$ (i.e., variance ratio in the sire model after removing dam variance from error variance, following VanRaden and Wiggans, 1991). It is worth noting that corrections to avoid double-counting due to pedigree information can possibly be ignored without a considerable effect on the evaluations.

Descriptive statistics for MACE EBV used in the analysis are shown in Table 1. Daughter equivalents (DE) were calculated following the work of VanRaden and Wiggans (1991). Total DE for an individual $\left(\mathrm{DE}_{\text {anim }}\right)$ is the sum of $\mathrm{DE}$ from parent average (PA; $\left.\mathrm{DE}_{\mathrm{PA}}\right)$, own yield $\left(\mathrm{DE}_{\text {yield }}\right)$, and progeny adjusted for mates $\left(\Sigma \mathrm{DE}_{\text {prog-mate }}\right)$ :

$$
\mathrm{DE}_{\text {anim }}=\mathrm{DE}_{\mathrm{PA}}+\mathrm{DE}_{\text {yield }}+\Sigma \mathrm{DE}_{\text {prog-mat }} \text {. }
$$

Each animal's reliability $\left(\operatorname{Rel}_{\text {anim }}\right)$ is calculated from $\mathrm{DE}_{\text {anim }}$ :

$$
\operatorname{Rel}_{\text {anim }}=\mathrm{DE}_{\text {anim }} /\left(\mathrm{DE}_{\text {anim }}+k_{d}\right),
$$

where $k_{d}$ is a variance ration calculated as $\left(4-2 \mathrm{~h}^{2}\right) / \mathrm{h}^{2}$. Thus, the previous equation can be reversed to calculate $\mathrm{DE}_{\text {anim }}$ from $\mathrm{Rel}_{\text {anim }}$ :

$$
\mathrm{DE}_{\text {anim }}=k_{d}\left(\operatorname{Rel}_{\text {anim }}\right) /\left(1-\operatorname{Re}_{\text {anim }}\right) .
$$

Multi-Step GBLUP Models. Consider the following model:

$$
\mathbf{y}=1 \mu+\mathbf{Z g}+\mathbf{e},
$$

where $\mathbf{y}$ is a vector of de-regressed evaluations of genotyped animals, $\mathbf{1}$ is a vector of ones, $\mu$ is the overall mean, $\mathbf{Z}$ is a design matrix that assigns records to direct genomic breeding values (DGV), $\mathrm{g}$ is a vector of DGV to be estimated, and $\mathbf{e}$ is a vector of random residual effects. It was assumed that $\mathbf{g} \sim N\left(\mathbf{0}, \mathbf{G} \sigma_{g}^{2}\right)$, where $\mathbf{G}$ is the genomic relationship matrix based on SNP marker genotypes and $\sigma_{g}^{2}$ is the additive genetic variance; and $\mathbf{e} \sim N\left(\mathbf{0}, \mathbf{R} \sigma_{e}^{2}\right)$, where $\mathbf{R}$ is a diagonal matrix containing weights based on the reliability of the breeding values and $\sigma_{e}^{2}$ is the residual variance. Here, DGV were estimated with $20 \%$ weight $(\delta)$ for the expected additive relationship matrix (A) when combined with the genomic relationship matrix. The weight of $20 \%$ is currently used in Canadian national genomic evaluations (Van Doormaal et al., 2009) to consider the polygenic effects not accounted for by the genomic markers. Thus, the model becomes

$$
\mathbf{y}=\mathbf{1} \mu+\mathbf{Z g}_{\delta}+\mathbf{e},
$$

where $\mathbf{g}_{\delta} \sim N\left(\mathbf{0}, \mathbf{G}_{\delta} \sigma_{g_{\delta}}^{2}\right)$, where $\mathbf{G}_{\delta}$ is a combined relationship matrix, $\mathbf{G}_{\delta}=\delta \mathbf{A}_{22}+(1-\delta) \mathbf{G}$. 
Blending. The blending approach used to generate GEBV was as follows:

$$
\begin{aligned}
\mathrm{GEBV} & =\mathrm{w}_{1} \mathrm{DGV}+\mathrm{w}_{2} \mathrm{EST}, \\
\mathrm{w}_{1} & =\frac{\operatorname{Rel}_{\mathrm{DGV}}}{\operatorname{Rel}_{\mathrm{DGV}}+\operatorname{Rel}_{\mathrm{EST}}}, \\
\mathrm{w}_{2} & =\frac{\operatorname{Rel}_{\mathrm{EST}}}{\operatorname{Rel}_{\mathrm{EST}}+\operatorname{Rel}_{\mathrm{DGV}}},
\end{aligned}
$$

where EST is either EBV (for training population animals) or PA (for validation population animals) from traditional BLUP and Rel is the reliability of estimates.

\section{Implementation}

Six different evaluations were performed to evaluate the effect of including MACE information in the domestic evaluation:

(1) Domestic traditional BLUP evaluation with domestic phenotypic and pedigree information $\left(\right.$ BLUP $\left._{\mathbf{D}}\right)$.

(2) Domestic evaluation with domestic phenotypic, genomic, and pedigree information using a single-step approach ( $\mathbf{s s G B L U P}_{\mathbf{D}}$ ).

(3) Domestic evaluation with domestic de-regressed EBV and genotypic information using a multistep approach $\left(\mathbf{m} \mathbf{s} \mathbf{G B L U} \mathbf{P}_{\mathbf{D}}\right)$.

(4) An evaluation where domestic information (for bulls that have or not MACE evaluations) of foreign bulls was analyzed with domestic phenotypic, genomic, and pedigree information in a single-step approach ( $\mathbf{s s G B L U P}_{\mathbf{J}}$ ).

(5) Domestic evaluation with domestic phenotypic, genomic, and pedigree information with integration of MACE information $\left(\mathrm{EBV}_{\mathrm{M}}\right)$ using a single-step method (ssGBLUP $\mathbf{P}_{\mathrm{IM}}$ ).

(6) Multi-national evaluation with domestic and MACE de-regressed EBV and genotypic information using a multi-step method (msGB$\left.\mathbf{L U P}_{\mathrm{MD}}\right)$.

For analyses using the single-step approach, different data sets were prepared: (1) a full data set containing all phenotypic records of cows from which EBV were calculated and de-regressed to be used as benchmarks in the evaluation process; (2) a reduced data set that included records of cows after deleting the last $4 \mathrm{yr}$ of observations; and for analysis with integration of MACE information, (3) a data set containing $\mathrm{EBV}_{\mathrm{M}}$ (obtained after corrections to avoid double-counting were applied) and associated reliabilities for foreign bulls. For the multi-step approach, de-regressed EBV of domestic and foreign genotyped bulls obtained from the 2013 evaluation carried out by CDN were used for prediction, and EBV obtained from the 2017 evaluation were used for validation. A total of 1,729 and 1,788 MACE bulls included in the analyses for MT and MSP, respectively, also had genomic information.

\section{Validation}

Genomic predictions (GEBV) obtained with the alternate prediction methods were compared based on their observed validation reliabilities and bias of predictions. The reliability of genomic predictions for the validation bulls were measured as squared correlations $\left(\mathbf{r}^{2}\right)$ between the predicted breeding values (obtained with the reduced data set) and DD (from the full data set). To assess bias of genomic predictions for each method, the following regression model was used (Mäntysaari et al., 2010):

$$
\mathbf{D D}_{\mathbf{C}}=\mathrm{b}_{0}+\mathrm{b}_{1} \mathbf{X}_{\mathbf{P}}+\mathbf{e}
$$

where $\mathbf{D D}_{\mathbf{C}}$ are de-regressed EBV obtained from complete data set of genotyped bulls with no daughters in the reduced data set, but with at least 50 daughters in the complete data set; $b_{0}$ is the intercept; $b_{1}$ is the linear regression coefficient indicating bias of predictions; $\mathbf{X}_{\mathbf{P}}$ is the bull's prediction obtained with the reduced data set; and $\mathbf{e}$ is the residual term.

\section{RESULTS AND DISCUSSION}

The calculation of an animal's breeding value aggregates information from the animal's own records (i.e., contributions due to records) and from records of its relatives (i.e., contributions due to relationships) (Misztal and Wiggans, 1988). When a national evaluation is based on integration of EBV from external sources (i.e., MACE EBV), avoiding double-counting of information becomes of utmost importance and a failure to do so could lead to biased predictions and inflated reliabilities (Vandenplas et al., 2017). In the present study, a total of 1,729 and 1,788 bulls with Interbull evaluations were available for the analysis of MT and MSP, respectively. Because double-counting of phenotypic contributions due to relationships can lead to inflation of predictions, corrections were applied to avoid possible doublecounting. From the total number of bulls with MACE EBV available, 100 and 109 bulls for MT and MSP, respectively, also had national (domestic) evaluations that were sent to Interbull and therefore contributed to 
MACE EBV calculation (Table 1). Double-counting of information was avoided by applying a discount factor on $\mathrm{EBV}_{\mathrm{M}}$ based on daughter equivalents from national and MACE EBV using Equations [5] and [6]. Although the correction for double-counting used in our study potentially adds an extra step to ssGBLUP, other studies have successfully implemented such corrections simultaneously in the analysis through a modification of the system of equations (Vandenplas et al., 2014, 2017; Colinet et al., 2018).

The number of genotyped bulls in the training population has a great effect on reliability of genomic predictions (VanRaden et al., 2009). Thus, the use of foreign (MACE) bulls in genetic evaluations becomes an appealing tool, as it has the potential to increase reliability compared with the use of domestic bulls only. Their inclusion will increase the size of the training population, which will likely lead to an increase in predictive ability of GBLUP. For msGBLUP, 6,189 and 6,321 domestic bulls were available for MT and MSP, respectively. With the inclusion of MACE bulls in the evaluation, the size of the training population for both traits increased about $28 \%$.

The use of genomic information for prediction increased $\mathrm{r}^{2}$ by 4.0 and $13.0 \%$ points for MT and MSP, respectively, for msGBLUP $_{D}$ compared with BLUP $_{D}$ (Table 3). When MACE bulls were included in the training population, msGBLUP $\mathrm{MD}_{\mathrm{MD}}$ led to an increase in $\mathrm{r}^{2}$ of 4.8 and $15.4 \%$ points for MT and MSP, respectively, compared with $\mathrm{BLUP}_{\mathrm{D}}$. Although reliabilities of MACE EBV were, on average, lower than those for domestic EBV (Table 2), adding this extra information in the training population still helped increase reliabilities, which underlines the importance of training population size and amount of information available.

The availability of MACE EBV enables the exchange of information from several countries; however, several limitations are still associated with their use in the context of a national evaluation through a multi-step approach. For instance, in genetic evaluations, traditional EBV are regressed toward parent averages based on their associated reliabilities. Thus, the use of such EBV in genomic evaluations could lead to double-counting of information, shrinkage of the GEBV, and inaccuracies of predictions, which is especially true for EBV with low reliability (Garrick et al., 2009). The use of deregressed information is then viewed as an alternative response variable that could overcome such problems. However, although de-regression of domestic EBV is quite straightforward, steps for obtaining de-regressed MACE EBV can be complicated and potentially cause inaccuracies during evaluation. This might be so because the parent average used for de-regression of an animal's EBV in the national evaluation may not match that used by Interbull for calculations of MACE EBV (Sargolzaei and Chesnais, 2014). Further complications arise when multi-trait evaluations are used (Schaeffer 2001), which is especially true when each country uses a different number of traits (Vandenplas and Gengler, 2015). Moreover, when MACE EBV of a bull is considered official but it greatly deviates from its national EBV, inflation may arise due to the fact that EBV of that bull's close relatives (i.e., progeny, cows, bulls without international EBV) are no longer in agreement with the bull's official breeding value (Vandenplas and Gengler, 2015). All these might help explain the very small to nonexistent improvements observed on regression coefficients (bias) of genomic predictions obtained with msGBLUP ${ }_{\mathrm{MD}}$ compared with msGBLUP $_{\mathrm{D}}$ (Table $3)$.

As expected, the use of a single-step approach led to higher reliabilities of genomic predictions. An increase of 5.2 and $6.3 \%$ for MT and MSP, respectively, was observed when ssGBLUP ${ }_{D}$ was used compared with

Table 2. Descriptive statistics of EBV used for genomic evaluations using multi-step genomic BLUP for milking temperament (MT) and milking speed (MSP)

\begin{tabular}{|c|c|c|c|c|c|c|c|c|}
\hline \multirow[b]{3}{*}{ Trait } & \multirow{2}{*}{\multicolumn{2}{|c|}{ Validation }} & \multicolumn{6}{|c|}{ Training } \\
\hline & & & \multicolumn{2}{|c|}{$\mathrm{MACE}^{1}$} & \multicolumn{2}{|c|}{ Domestic $^{2}$} & \multicolumn{2}{|c|}{$\mathrm{All}^{3}$} \\
\hline & $\begin{array}{l}\text { No. of } \\
\text { bulls }{ }^{4}\end{array}$ & $\begin{array}{c}\operatorname{Rel}_{\mathrm{PA}}{ }^{5} \\
(\%)\end{array}$ & $\begin{array}{c}\text { No. of } \\
\text { bulls }\end{array}$ & $\begin{array}{c}\operatorname{Rel}_{\mathrm{dEBV}}{ }^{6} \\
(\%)\end{array}$ & $\begin{array}{l}\text { No. of } \\
\text { bulls }\end{array}$ & $\begin{array}{c}\operatorname{Rel}_{\mathrm{dEBV}} \\
(\%)\end{array}$ & $\begin{array}{c}\text { No. of } \\
\text { bulls }\end{array}$ & $\begin{array}{c}\operatorname{Rel}_{\mathrm{dEBV}} \\
(\%)\end{array}$ \\
\hline MT & 554 & 25.8 & 1,729 & 36.1 & 6,189 & 74.4 & 7,918 & 66.0 \\
\hline MSP & 554 & 26.3 & 1,788 & 54.5 & 6,321 & 76.1 & 8,109 & 71.3 \\
\hline
\end{tabular}

${ }^{1}$ Bulls with multi-trait across-country evaluation (MACE) EBV included in the training population.

${ }^{2}$ Bulls with domestic EBV included in the training population.

${ }^{3}$ All bulls included in the training population.

${ }^{4}$ Only domestic bulls with at least 50 daughters in the 2017 evaluation were considered in validation population.

${ }^{5} \mathrm{Re}_{\mathrm{PA}}=$ average reliability of the parent averages.

${ }^{6} \mathrm{Rel}_{\mathrm{dEBV}}=$ average reliability of de-regressed EBV used in the training population of multi-step genomic BLUP. 
Table 3. Observed reliabilities $\left(\mathrm{r}^{2}\right)$ of genomic predictions and regression coefficient $\left(\mathrm{b}_{1}\right)$ of de-regressed EBV on genomic predictions for 554 genotyped bulls, with at least 50 daughters, for milking temperament (MT) and milking speed (MSP) for different genomic evaluations

\begin{tabular}{|c|c|c|c|c|c|c|c|c|c|c|c|c|}
\hline Trait & \multicolumn{2}{|c|}{$\mathrm{BLUP}_{\mathrm{D}}^{1}$} & \multicolumn{2}{|c|}{$\operatorname{ssGBLUP}_{\mathrm{D}}^{2}$} & \multicolumn{2}{|c|}{$\operatorname{msGBLUP}_{\mathrm{D}}{ }^{3}$} & \multicolumn{2}{|c|}{$\operatorname{ssGBLUP}_{\mathrm{J}}^{4}$} & \multicolumn{2}{|c|}{$\operatorname{ssGBLUP}_{\mathrm{IM}}^{5}$} & \multicolumn{2}{|c|}{$\operatorname{msGBLUP}_{\mathrm{MD}}{ }^{6}$} \\
\hline MSP & 0.975 & 0.219 & 0.917 & 0.412 & 1.086 & 0.349 & 0.957 & 0.417 & 0.983 & 0.426 & 1.104 & 0.373 \\
\hline
\end{tabular}

${ }^{1}$ Domestic traditional BLUP evaluation with domestic phenotypic and pedigree information.

${ }^{2}$ Domestic evaluation with domestic phenotypic, genomic, and pedigree information using a single-step approach.

${ }^{3}$ Domestic evaluation with domestic de-regressed EBV and genotypic information using a multi-step approach.

${ }^{4}$ Joint evaluation where all available information (domestic and multi-national phenotypic, genomic, and pedigree) were used in a single-step approach.

${ }^{5}$ Domestic evaluation with domestic phenotypic, genomic, and pedigree information with integration of multi-trait across-country evaluation (MACE) information ( $\left.\mathrm{EBV}_{\mathrm{IM}}\right)$ using a single-step method.

${ }^{6}$ Multi-national evaluation with domestic and MACE de-regressed EBV and genotypic information using a multi-step method.

msGBLUP $_{\mathrm{D}}$, and 9.6 and $19.3 \%$ when compared with BLUP $_{D}$ (Table 3). The highest reliabilities were obtained with ssGBLUP ${ }_{\text {IM }}$, which used integration of MACE information into the domestic evaluation, while correcting for double-counting. Integration also improved bias of genomic predictions (regression coefficient values were closer to 1.00). Although overestimation of predictions was already minimum for pedigree-based evaluation for MSP $\left(b_{1}=0.975\right)$, by using a single-step approach, regression coefficients approached 1 (Table 3). However, there was an underestimation of genomic predictions for the same trait when a multi-step approach was adopted. For MT, less inflated predictions were observed with integration of MACE EBV into domestic evaluation. Absolute deviations of the regression coefficients from 1 for MT went from 0.46 with BLUP $_{D}$ to 0.42 msGBLUP $_{\mathrm{MD}}$. When integration of MACE information was implemented through a single-step approach (ssGBLUP $\mathrm{IM}_{\mathrm{IM}}$ ), the absolute deviation from 1 decreased by half compared with msGBLUP MD $(0.21)$.

In the present study, ssGBLUP $_{\mathrm{J}}$ can be considered as a reference. This is a hypothetical scenario where all available information (i.e., phenotypes, pedigree, and genotypes) in the national database, which also includes domestic information of foreign bulls, is used simultaneously in a joint evaluation. Given that ssGBLUP $_{\text {IM }}$ uses all domestic information available while incorporating external information of foreign bulls (i.e., MACE EBV), the slightly increased reliabilities and reduced inflation of genomic predictions obtained with this method, compared with ssGBLUP ${ }_{\mathrm{J}}$, showed that integration of MACE information through a single-step approach was successful. These findings were in agreement with previous studies that also attempted to implement an integration method using a unified approach (Vandenplas et al., 2014, 2017).

Besides the importance of the size of the training data set, genetic relationships between its individuals and young bulls whose breeding values are to be predicted, are also a critical factor affecting the accuracy of genomic breeding values (Habier et al., 2010; Clark et al., 2012). The gains obtained with the inclusion of MACE bulls in the present study could be partially explained by their genetic relationship with those from the validation data set. When MACE bulls are not included in the analysis, the estimation of breeding values is largely dependent on distant relatives, from which lower reliabilities were obtained. However, when MACE bulls are included, given the close relationship with animals from validation data set, more weight is placed on their information, instead of distantly related animals, and hence an increase in reliability is expected. Thus, given the importance of those animals for breeding programs, this study emphasizes the need for a methodology that properly incorporates MACE information into a domestic evaluation.

To our knowledge, no studies have been previously published on the use of MACE information in domestic evaluations using a single-step approach for dairy cattle in Canada and United States using real data. The proposed method greatly improved reliabilities and bias of genomic predictions for the studied traits compared with a multi-step approach, which was expected given the fact that ssGBLUP uses all available information simultaneously. Nonetheless, improvements obtained with ssGBLUP $_{\text {IM }}$ compared with ssGBLUP $_{\mathrm{J}}$ shows that, even though the amount of MACE information was small, it had a favorable effect on genomic predictions, and therefore, integration should be considered. Our study highlights the importance of the method used for prediction of breeding values, and also the importance of the size and structure of the training population used for genomic evaluations. Using MACE EBV corrected for double-counting due to domestic information in the BLUP90MBE software is straightforward and only requires an independent script (i.e., 
to perform corrections as in Equations [5] and [6] before running BLUP90MBE), instead of changing the system of equations, which can considerably increase computing time. For a bull with high reliability, the amount of its "own" information (i.e., contribution from progeny) used to calculate its EBV is usually larger compared with contribution from relatives (Calus et al., 2016). Thus, avoiding double-counting of information is especially important for evaluations based on bulls with lower reliabilities (e.g., bulls with a small number of progeny).

We expect that the analyses described here will be more beneficial for countries where the number of proven domestic bulls for genomic evaluation is limited. For instance, an improvement of 4 percentage points in reliability was observed with the inclusion of MACE information for genomic evaluation of udder depth in US Holsteins using a population of 105,116 domestic genotyped animals (Shogo Tsuruta, unpublished data). However, when external information was integrated using a similar methodology in a simulated population with genomic information of only 1,000 domestic animals, reliabilities doubled when compared with a scenario where genomic evaluation was performed considering only domestic information (Andonov et al., 2017).

\section{CONCLUSIONS}

The use of a single-step approach with integration of MACE information provides an alternative to the current method used in Canadian genomic evaluations. The proposed method leads to better results compared with the multi-step approach, with less bias and more reliable predictions. Moreover, because phenotypes, genotypes, pedigree, and MACE EBV are evaluated simultaneously, the single-step approach overcomes the limitations that arise from de-regressing MACE EBV for use in a multi-step approach. Although in our study one extra step was added to perform the correction of MACE EBV for double-counting based on modifications of previously proposed methods, further developments will allow corrections simultaneously in the single-step analysis in a more efficient way.

\section{ACKNOWLEDGMENTS}

This research was supported in main part by Agriculture and Agri-Food Canada (Ottawa, ON, Canada) and by additional contributions from Dairy Farmers of Canada (Ottawa, ON, Canada), the Canadian Dairy Network (Guelph, ON, Canada), and the Canadian Dairy Commission (Ottawa, ON, Canada) under the Agri-Science Clusters Initiative. The first author is also grateful to CAPES (Brazilian Federal Agency for Support and Evaluation of Graduate Education, Ministry of Education, Brasilia, Brazil) for financial support.

\section{REFERENCES}

Aguilar, I., I. Misztal, D. L. Johnson, A. Legarra, S. Tsuruta, and T. J. Lawlor. 2010. Hot topic: a unified approach to utilize phenotypic, full pedigree, and genomic information for genetic evaluation of Holstein final score. J. Dairy Sci. 93:743-752. https://doi.org/10 .3168/jds.2009-2730.

Andonov, S., D. A. L. Lourenco, B. O. Fragomeni, Y. Masuda, I. Pocrnic, S. Tsuruta, I. Misztal, T. J. Lawlor, Z. Liu, R. Reents, C. Schrooten, F. Seefried, G. Su, D. Abernethy, and R. Spelman. 2017. Accuracy of breeding values in small genotyped populations using different sources of external information-A simulation study. J. Dairy Sci. 100:395-401. https://doi.org/10.3168/jds.2016-11335.

Bonaiti, B., and D. Boichard. 1995. Accounting for foreign information in genetic evaluation. Interbull Bull. 11.

Calus, M. P. L., J. Vandenplas, J. ten Napel, and R. F. Veerkamp. 2016. Validation of simultaneous deregression of cow and bull breeding values and derivation of appropriate weights. J. Dairy Sci. 99:6403-6419. https://doi.org/10.3168/jds.2016-11028.

Christensen, O. F., and M. S. Lund. 2010. Genomic prediction when some animals are not genotyped. Genet. Sel. Evol. 42:2. https:// doi.org/10.1186/1297-9686-42-2.

Clark, S. A., J. M. Hickey, H. D. Daetwyler, and J. H. van der Werf. 2012. The importance of information on relatives for the prediction of genomic breeding values and the implications for the makeup of reference data sets in livestock breeding schemes. Genet. Sel. Evol. 44:4. https://doi.org/10.1186/1297-9686-44-4.

Colinet, F. G., J. Vandenplas, S. Vanderick, H. Hammami, R. R. Mota, A. Gillon, X. Hubin, C. Bertozzi, and N. Gengler. 2018. Bayesian single-step genomic evaluations combining local and foreign information in Walloon Holsteins. Animal 12:898-905.

Garrick, D. J., J. F. Taylor, and R. L. Fernando. 2009. Deregressing estimated breeding values and weighting information for genomic regression analyses. Genet. Sel. Evol. 41:55. https://doi.org/10 $.1186 / 1297-9686-41-55$.

Gengler, N., and S. Vanderick. 2008. Bayesian inclusion of external evaluations into a national evaluation system: Application to milk production traits. Interbull Bull. 38:70-74.

Gianola, D., and R. L. Fernando. 1986. Bayesian methods in animal breeding theory. J. Anim. Sci. 63:217-244.

Habier, D., J. Tetens, F.-R. Seefried, P. Lichtner, and G. Thaller. 2010. The impact of genetic relationship information on genomic breeding values in German Holstein cattle. Genet. Sel. Evol. 42:5. https: //doi.org/10.1186/1297-9686-42-5.

Legarra, A., I. Aguilar, and I. Misztal. 2009. A relationship matrix including full pedigree and genomic information. J. Dairy Sci. 92:4656-4663. https://doi.org/10.3168/jds.2009-2061.

Legarra, A., J. K. Bertrand, T. Strabel, R. L. Sapp, J. P. Sanchez, and I. Misztal. 2007. Multi-breed evaluations for beef cattle. J. Anim. Breed. Genet. 124:286-295. https://doi.org/10.1111/j.1439 $-0388.2007 .00671 . x$.

Legarra, A., O. F. Christensen, I. Aguilar, and I. Misztal. 2014. Single step, a general approach for genomic selection. Livest. Sci. 166:5465. https://doi.org/10.1016/j.livsci.2014.04.029.

Lourenco, D. A. L., S. Tsuruta, B. O. Fragomeni, Y. Masuda, I. Aguilar, A. Legarra, S. Miller, D. Moser, and I. Misztal. 2018. Singlestep genomic BLUP for national beef cattle evaluation in US From initial developments to final implementation. In Proc. 11th WCGALP, Auckland, New Zealand. World Congress on Genetics Applied to Livestock Production Digital Archives. 11.495. http:// www.wcgalp.org/proceedings/2018.

Mäntysaari, E. A., Z. Liu, and P. VanRaden. 2010. Interbull validation test for genomic evaluations. Interbull Bull. 41:17.

Misztal, I., I. Aguilar, A. Legarra, and T. J. Lawlor. 2010. Choice of parameters for single-step genomic evaluation for type. J. Dairy Sci. 93(Suppl. 1):533. (Abstr.) 
Misztal, I., and G. R. Wiggans. 1988. Approximation of prediction error variance in large-scale animal models. J. Dairy Sci. 71:27-32. https://doi.org/10.1016/S0022-0302(88)79976-2.

Quaas, R. L., and Z. Zhang. 2001. Incorporating external information in multi-breed genetic evaluation. J. Anim. Sci. 79(Suppl. 1):342. (Abstr.)

Quaas, R. L., and Z. Zhang. 2006. Multi-breed Genetic Evaluation in the US Beef Cattle Context: Methodology. Communication 24-12 in CD of 8th World Congr. Appl. Livest. Prod., Belo Horizonte, Brazil.

Sargolzaei, M., and J. Chesnais. 2014. Impact of de-regression of MACE proofs on bias and accuracy of genomic breeding values. Research Report to DCGBC, February 2014.1-19.

Schaeffer, L. R. 1994. Multiple-country comparison of dairy sires. J. Dairy Sci. 77:2671-2678. https://doi.org/10.3168/jds.S0022 -0302(94)77209-X

Schaeffer, L. R. 2001. Multiple trait international bull comparisons. Livest. Prod. Sci. 69:145-153. https://doi.org/10.1016/S0301 $-6226(00) 00255-4$.

Tsuruta, S., I. Misztal, I. Aguilar, and T. J. Lawlor. 2011. Multiple-trait genomic evaluation of linear type traits using genomic and phenotypic data in US Holsteins. J. Dairy Sci. 94:4198-4204. https://doi.org/10.3168/jds.2011-4256.

Tsuruta, S., I. Misztal, and I. Stranden. 2001. Use of the preconditioned conjugate gradient algorithm as a generic solver for mixedmodel equations in animal breeding applications. J. Anim. Sci. 79:1166-1172.

Van Doormaal, B. J., G. J. Kistemaker, P. G. Sullivan, M. Sargolzaei, and F. S. Schenkel. 2009. Canadian implementation of genomic evaluations. Interbull Bull. 40:214-218.

Vandenplas, J., F. G. Colinet, and N. Gengler. 2014. Unified method to integrate and blend several, potentially related, sources of infor- mation for genetic evaluation. Genet. Sel. Evol. 46:59. https://doi .org/10.1186/s12711-014-0059-3.

Vandenplas, J., and N. Gengler. 2015. Strategies for comparing and combining different genetic and genomic evaluations: A review. Livest. Sci. 181:121-130. https://doi.org/10.1016/j.livsci.2015.09 .012 .

Vandenplas, J., M. Spehar, K. Potocnik, N. Gengler, and G. Gorjanc. 2017. National single-step genomic method that integrates multinational genomic information. J. Dairy Sci. 100:465-478. https:// doi.org/10.3168/jds.2016-11733.

VanRaden, P. M. 2008. Efficient methods to compute genomic predictions. J. Dairy Sci. 91:4414-4423. https://doi.org/10.3168/jds .2007-0980.

VanRaden, P. M., M. E. Tooker, J. R. Wright, C. Sun, and J. L. Hutchison. 2014. Comparison of single-trait to multi-trait national evaluations for yield, health, and fertility. J. Dairy Sci. 97:79527962. https://doi.org/10.3168/jds.2014-8489.

VanRaden, P. M., C. P. Van Tassell, G. R. Wiggans, T. S. Sonstegard, R. D. Schnabel, J. F. Taylor, and F. S. Schenkel. 2009. Invited review: Reliability of genomic predictions for North American Holstein bulls. J. Dairy Sci. 92:16-24. https://doi.org/10.3168/jds .2008-1514.

VanRaden, P. M., and G. R. Wiggans. 1991. Derivation, calculation, and use of national animal model information. J. Dairy Sci. $74: 2737-2746$.

Vitezica, Z. G., I. Aguilar, I. Misztal, and A. Legarra. 2011. Bias in genomic predictions for populations under selection. Genet. Res. (Camb.) 93:357-366. https://doi.org/10.1017/ S001667231100022X 